Supporting Information for

\title{
Rapid Fabrication of High Entropy Ceramic Nanomaterials for Catalytic Reactions
}

\author{
Jie Xiang Yang ${ }^{1,4 \#}$ Bai-Hao Dai ${ }^{1 \#}$ Ching-Yu Chiang ${ }^{3 \#}$ I-Chia Chiu $^{1}$ Chih-Wen Pao ${ }^{3}$ Sheng-Yuan Lu ${ }^{1}$ I-Yu Tsao \\ Shou-Tai Lin ${ }^{5}$ Ching-Ting Chiu ${ }^{1}$ Jien-Wei Yeh ${ }^{2,6}$ Pai-Chun Chang $^{7}$ Wei-Hsuan Hung ${ }^{1,2 *}$ \\ ${ }^{1}$ Institute of Materials Science and Engineering, National Central University, \\ No. 300 Jhong-da Rd., Jhongli City 320, Taoyuan County, Taiwan, ROC \\ ${ }^{2}$ High Entropy Materials Center,101, Sec. 2, Kuang-Fu Road, Hsinchu 30013, Taiwan \\ ${ }^{3}$ National Synchrotron Radiation Research Center (NSRRC) \\ No. 101, Xin'an Road, East District, Hsinchu City, 300 \\ ${ }^{4}$ Department of Materials Science and Engineering, Feng Chia University, \\ No. 100 Wenhwa Rd., Seatwen, Taichung, Taiwan 40724, R.O.C. \\ ${ }^{5}$ Department of Photonics, Feng Chia University, \\ No. 100 Wenhwa Rd., Seatwen, Taichung, Taiwan 40724, R.O.C. \\ ${ }^{6}$ Department of Material Science and Engineering, National Tsing Hua University, \\ 101, Sec. 2, Kuang-Fu Road, Hsinchu 30013, Taiwan. \\ ${ }^{7}$ Nano Ark Co. San Jose, California 95120, USA \\ *Email: hungwh@ncu.edu.tw \\ \#The authors contributed equally to this work.
}

Additionally, we also tested our CoCrFeNiAl HECs performance in a neutral solution $(\mathrm{Kpi} \mathrm{pH}=7)$, which also shows good activity on both the onset voltage and high current density in Figure S1.

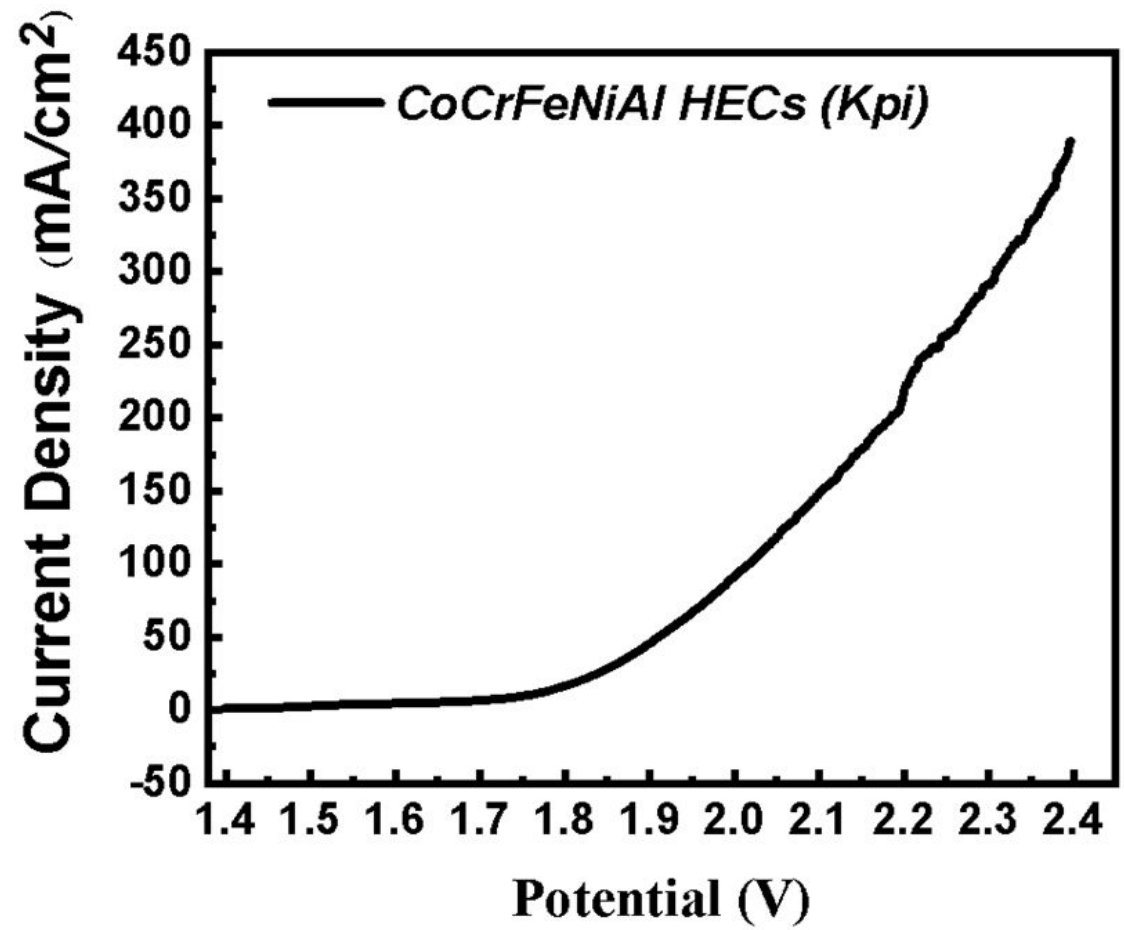

Figure S1. LSV curve of electrocatalytic activity of CoCrFeNiAl high-entropy ceramic nanoparticle catalyst electrode in a neutral solution (Kpi pH=7). 
In addition, the comparison of XRD spectrum of CoCrFeNiAl HECs before and after the reaction was shown in the supporting materials as Figure $\mathbf{S 2}$.
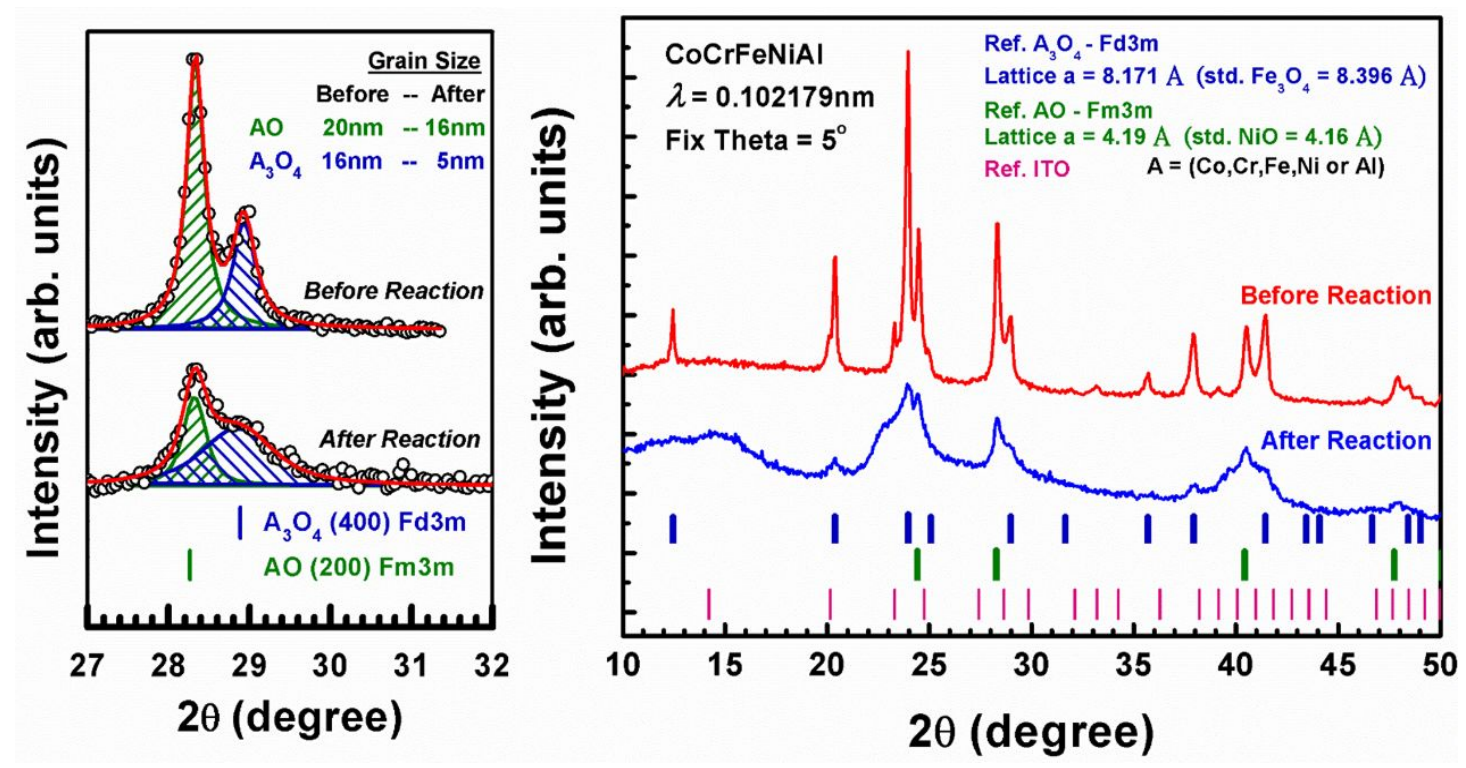

Figure S2. The comparison of XRD spectrum of CoCrFeNiAl HECs before and after reaction

Besides the ITO and Ni foam substrates, we successfully integrated the HECs NPs on the carbon cloth via PLMS shown in Figure S3.

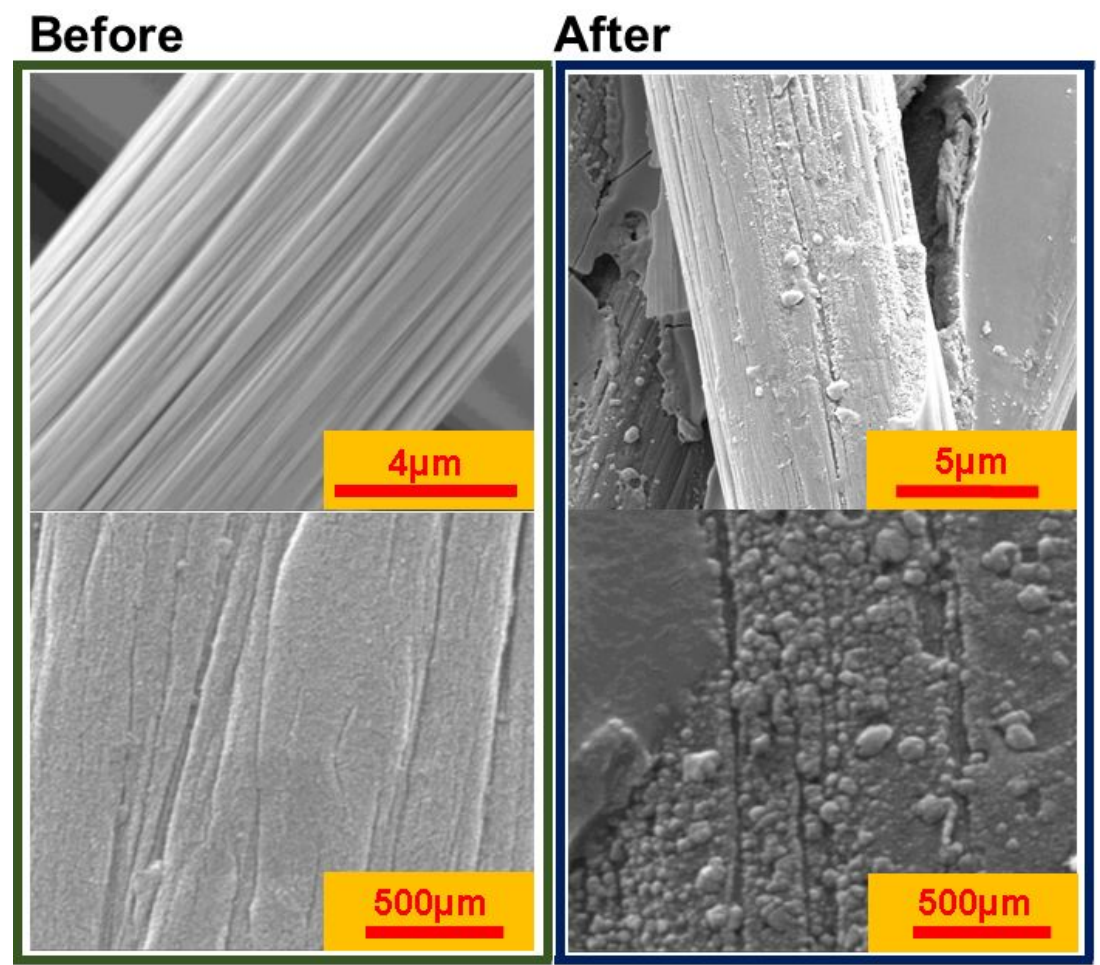

Figure S3. The HECs NPs were successfully on the surface of carbon cloth via PLMS method. The surface of carbon cloth (a) before (b) after PLMS 
Beside the nitrate precursors, we also adopted the other two different precursors, the chlorate and acetate successfully obtained the HECs by using these different precursors shown in Figure S4.

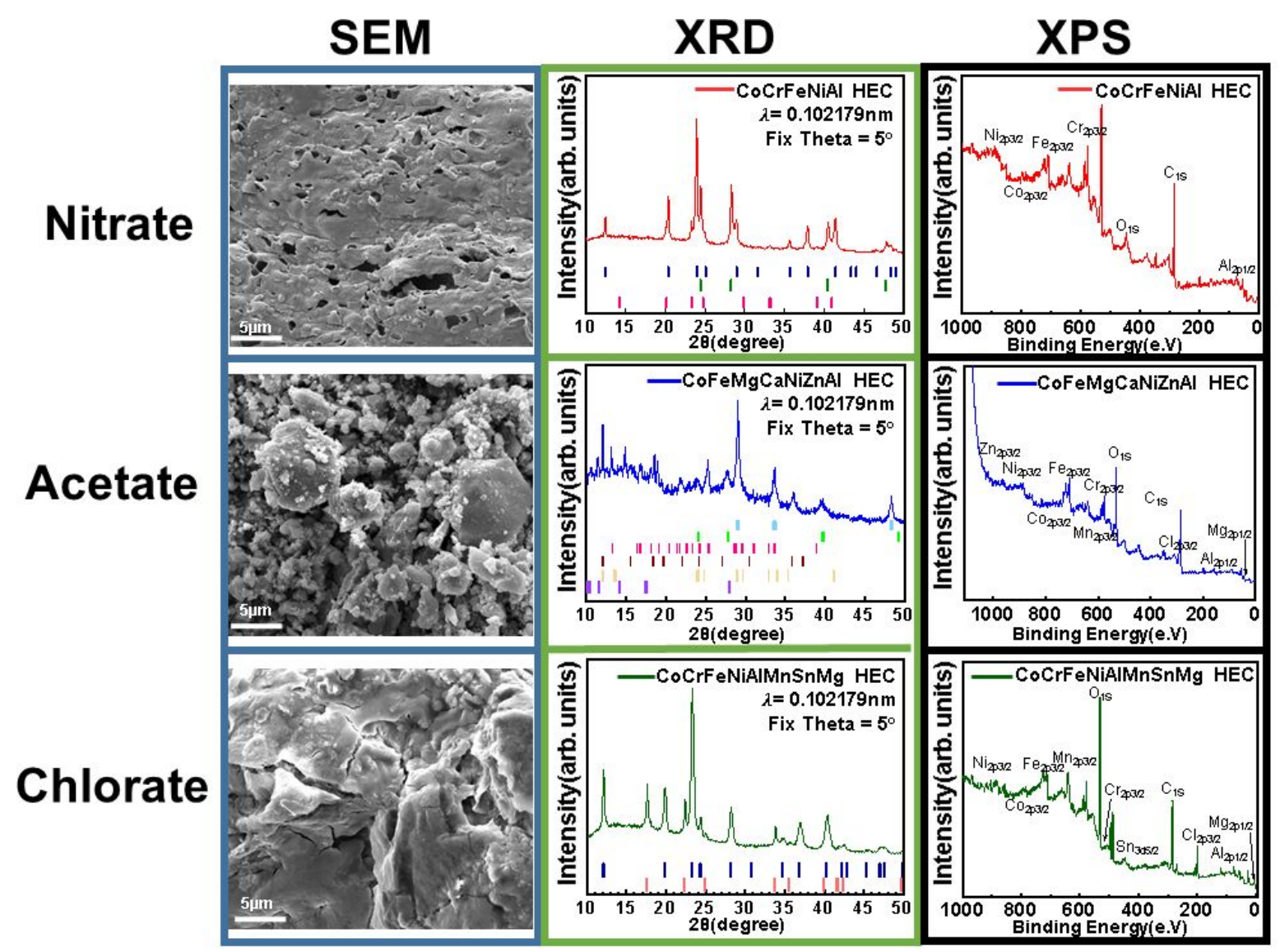

Figure S4. SEM, XRD, XPS of HECs fabricated from the nitrate, chlorate and acetate as precursors.

Furthermore, we also tested our $\mathrm{CoCrFeNiAl} \mathrm{HEC} \mathrm{under} \mathrm{much} \mathrm{higher} \mathrm{current} \mathrm{density}$ $\left(400 \mathrm{~mA} / \mathrm{cm}^{2}\right)$, which is a standard current density requirement for the industrial application shown in Figure S5. Our CoCrFeNiAl HECs exhibited extraordinary performance under such high current density for more than $150 \mathrm{hrs}$. 


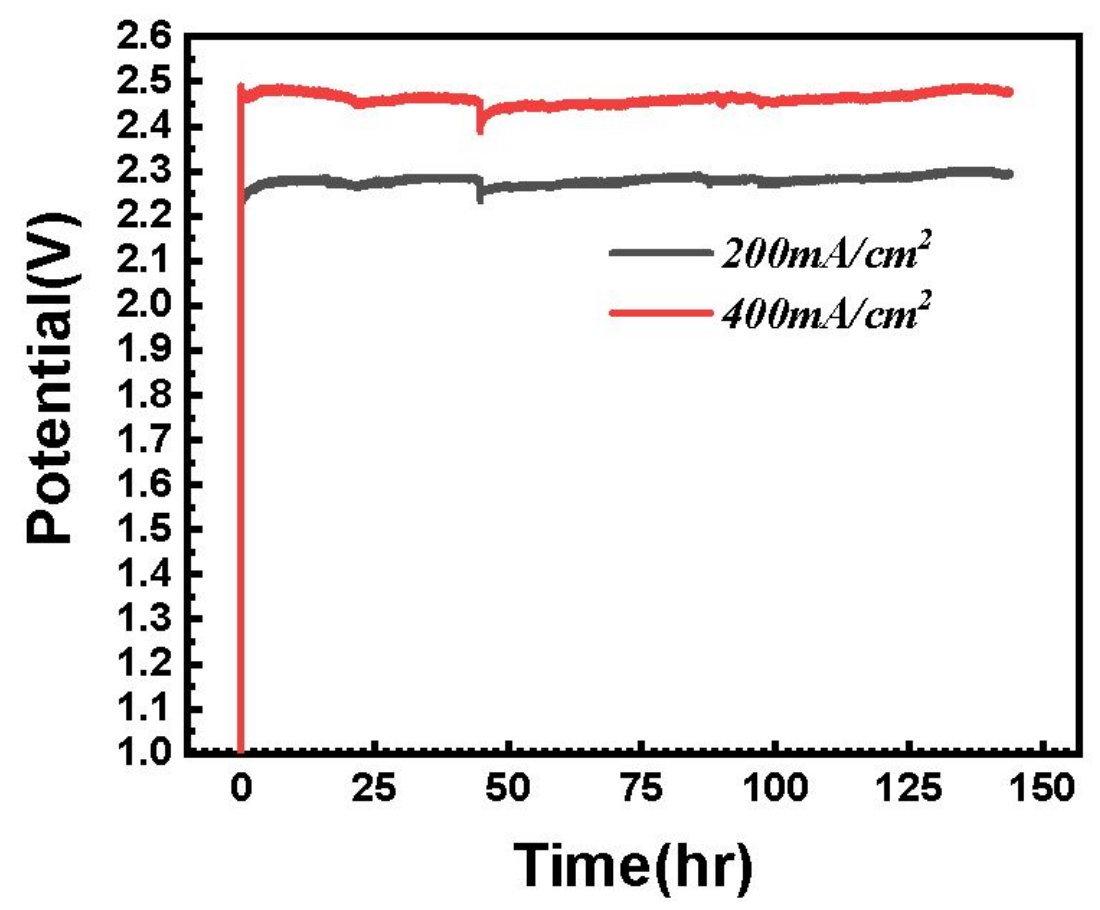

Figure S5. The stability test of CoCrFeNiAl HECs under the current density of 200 $\mathrm{mA} / \mathrm{cm}^{2}$ and $400 \mathrm{~mA} / \mathrm{cm}^{2}$

In order to further verify the uniformity of our HECs, we carried out the EDS mapping by the higher resolution STEM, which is also provided in Figure S6.
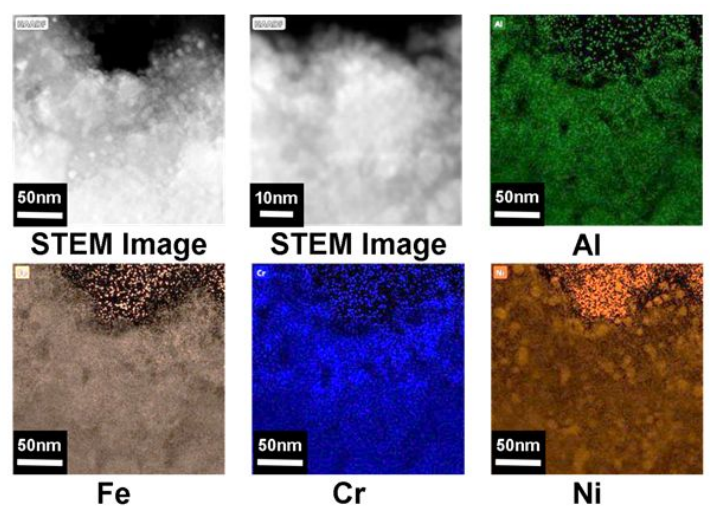

Al

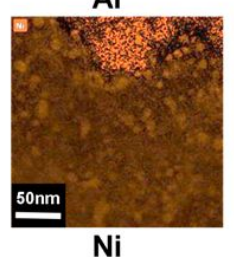

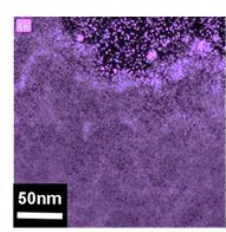

Co

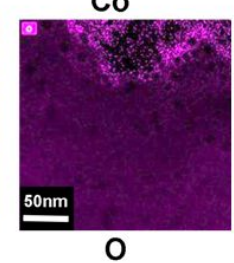

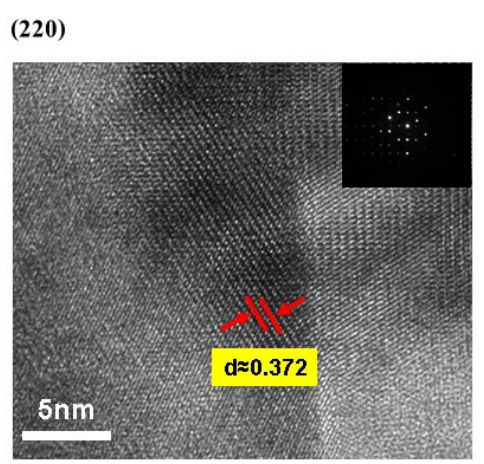

Figure S6. The high resolution STEM image and its EDS mapping

To verify the ratio of each element in our HECs after PLMS treatment, we performed the inductively coupled plasma-mass spectrometer (ICP) measurement. The result shows that experimental data has a good agreement with the designed ratio shown as a 
table below, which implies the PLMS possessed a good capability of the fidelity of the designed ratio of elements. (obtained in supporting materials Table 1)

\begin{tabular}{|c|c|c|c|c|}
\hline & $\begin{array}{c}\text { Designed } \\
\text { ratio(molar\%) }\end{array}$ & $\begin{array}{c}\text { Designed } \\
\text { ratio(wt\%) }\end{array}$ & $\begin{array}{c}\text { experimental } \\
\text { data(molar\%) }\end{array}$ & $\begin{array}{c}\text { experimental } \\
\text { data(wt\%) }\end{array}$ \\
\hline $\mathrm{Al}$ & 0.21 & 0.114048 & 0.21 & 0.114175 \\
\hline $\mathrm{Co}$ & 0.18 & 0.211345 & 0.19 & 0.214494 \\
\hline $\mathrm{Cr}$ & 0.12 & 0.119881 & 0.12 & 0.127061 \\
\hline $\mathrm{Fe}$ & 0.16 & 0.178827 & 0.17 & 0.186088 \\
\hline $\mathrm{Ni}$ & 0.33 & 0.375898 & 0.31 & 0.358182 \\
\hline
\end{tabular}

Table 1. The ICP measurement of CoCrFeNiAl HECs powder. 